\title{
Binding of pig sperm receptor in the zona pellucida to the boar sperm acrosome
}

\author{
N. Yonezawa ${ }^{1}$, Y. Hatanaka ${ }^{2}$, H. Takeyama ${ }^{1}$ and M. Nakano ${ }^{1,2}$ \\ ${ }^{1}$ Department of Chemistry, Faculty of Science, and ${ }^{2}$ Graduate School of Science and Technology, \\ Chiba University, 1-33 Yayoi-cho, Inage-ku, Chiba-shi, Chiba 263, Japan
}

\begin{abstract}
Pig zona pellucida (ZP) contains three families of glycoproteins: PZP2, PZP $3 \alpha$ and PZP $3 \beta$. $\mathrm{PZP} 3 \alpha$ mediates the binding of the $\mathrm{ZP}$ to spermatozoa. In this study, the binding site of pig ZP on boar spermatozoa and the zona-binding proteins of boar spermatozoa were studied using chemically modified zona glycoproteins or anti-pig ZP antiserum. Endo- $\beta$-galactosidase-digested PZP $3 \alpha$ (E $\beta$ G-PZP $3 \alpha$ ), which is deficient in sulfated $N$-acetylpolylactosamine, as well as solubilized $\mathrm{ZP}$, bound to the acrosomal region of acrosome-damaged or partially acrosome-reacted spermatozoa. However, they did not bind to acrosome-intact or fully acrosome-reacted spermatozoa. Solubilized ZP did bind to the acrosomal cap released upon acrosome reaction. In western blot analyses, E $\beta$ G-PZP $3 \alpha$ bound to the sperm proteins with molecular masses similar to proacrosin-acrosin and the binding was inhibited by fucoidan and anti-pig acrosin antiserum. These results suggest that the binding site of solubilized pig ZP and E $\beta$ G-PZP $3 \alpha$ on spermatozoa is located mainly in the acrosomal matrix and on the membranous compartments in the acrosome and suggest that E $\beta$ G-PZP $3 \alpha$ binds to proacrosin-acrosin. The binding of E $\beta$ G-PZP $3 \alpha$ to proacrosinacrosin may be involved in the binding of the $\mathrm{ZP}$ to the acrosome of partially acrosome-reacted spermatozoa.
\end{abstract}

\section{Introduction}

The zona pellucida (ZP) surrounding mammalian oocytes plays an important role in species-specific recognition between gametes. Pig ZP components can be separated by gel filtration column chromatography into two main fractions of molecular mass $90 \mathrm{kDa}$ and $55 \mathrm{kDa}, \mathrm{PZP} 2$ and $\mathrm{PZP} 3$, respectively (Nakano et al, 1987). PZP2 is cleaved into $25 \mathrm{kDa}$ and $65 \mathrm{kDa}$ fragments at a specific site upon fertilization (Hatanaka et al, 1992), but its function in gamete recognition has not been determined. PZP3 displays sperm receptor activity and consists of two distinct polypeptides, PZP $3 \alpha$ and PZP3 $\beta$ (Hedrick and Wardrip, 1987; Yurewicz et al., 1987). Heterogeneities of PZP 3 in charge and size are due to the presence of sulfated $N$-acetyllactosamine repeats in acidic $N$ - and $O$-linked oligosaccharides. Separation of PZP $3 \alpha$ and PZP $3 \beta$ is successful only after removal of sulfated $\mathrm{N}$-acetylpolylactosamine by digestion with endo- $\beta$-galactosidase (Yurewicz et al, 1987). Therefore, in this study the purified PZP $3 \alpha$ and $\mathrm{PZP} 3 \beta$ following endo- $\beta$-galactosidase digestion are referred to as endo- $\beta$-galactosidase-digested PZP $3 \alpha$ (E $\beta \mathrm{G}-\mathrm{PZP} 3 \alpha$ ) and endo$\beta$-galactosidase-digested PZP $3 \beta$ (E $\beta$ G-PZP $3 \beta$ ), respectively. Among the endo- $\beta$-galactosidase-digested zona components, only E $\beta$ G-PZP $3 \alpha$ inhibits sperm-zona binding in a competition assay in vitro (Sacco et al., 1989). Although the partial amino acid sequences of E $\beta G-P Z P 3 \beta$ reported by Yurewicz et al. $(1987,1992)$ are very similar to the sequence of the mouse

Revised manuscript received 20 July 1994. sperm receptor ZP3, E $\beta G-P Z P 3 \beta$ does not display sperm receptor activity.

The acrosomal status of spermatozoa that bind to $\mathrm{ZP}$ varies according to species. Acrosome-reacted spermatozoa bind to ZP in guinea-pigs (Yanagimachi, 1981), whereas in mice and humans, acrosome-intact spermatozoa bind to $\mathrm{ZP}$, which then induces the acrosome reaction (Wassarman, 1988; Liu and Baker, 1990). In pigs the acrosomal status of spermatozoa that bind to the ZP has not been clarified. Solubilized pig ZP has also been reported to induce acrosome reaction in ejaculated spermatozoa, but the increase in the percentage of acrosomereacted spermatozoa is small (Berger et al., 1989). Jones et al. (1988) hypothesized that in pigs, partially acrosome-reacted spermatozoa bind firmly to the ZP and then undergo acrosome reaction. Thus, the mechanisms of sperm-zona binding and initiation of acrosome reaction may vary according to species.

In this study, we investigated the binding site of pig $\mathrm{ZP}$ on spermatozoa and the sperm proteins that bind to E $\beta \mathrm{G}-\mathrm{PZP} 3 \alpha$, which is deficient in sulfated $\mathrm{N}$-acetylpolylactosamine.

\section{Materials and Methods}

\section{Purification of zona glycoproteins}

Pig ZP was isolated from ovaries and then solubilized at $70^{\circ} \mathrm{C}$ for $30 \mathrm{~min}$ in distilled water as described by Nakano et al. (1990). Solubilized ZP was digested with endo- $\beta$-galactosidase (Seikagaku Corp., Tokyo) as described by Yurewicz et al. (1987) 


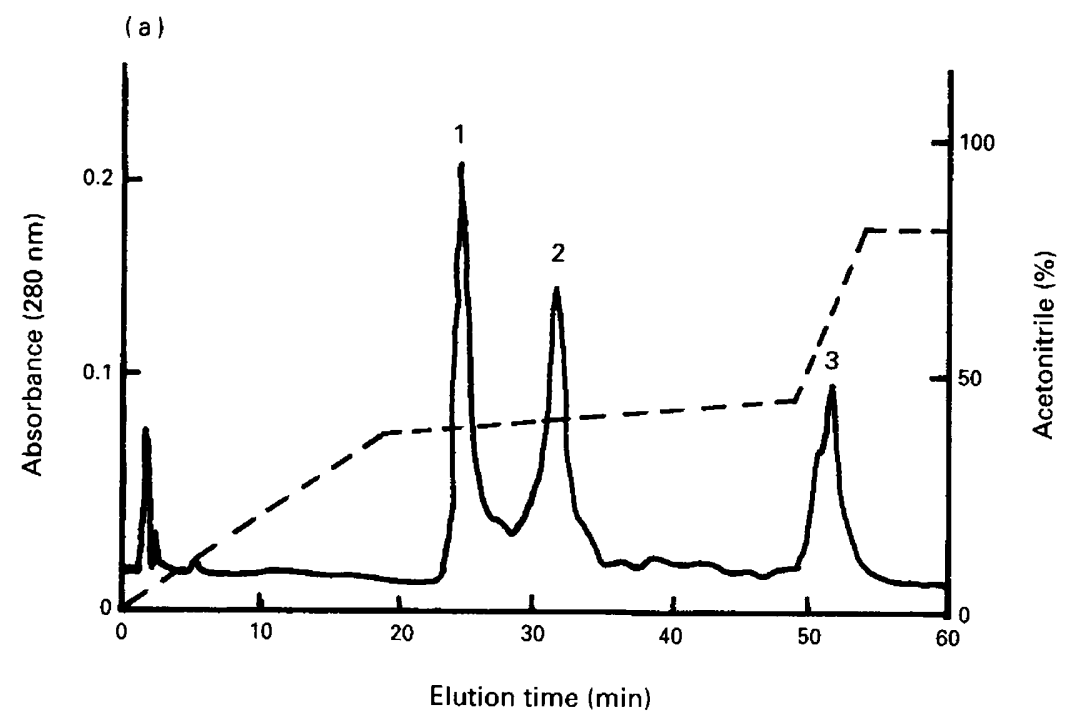

(b)

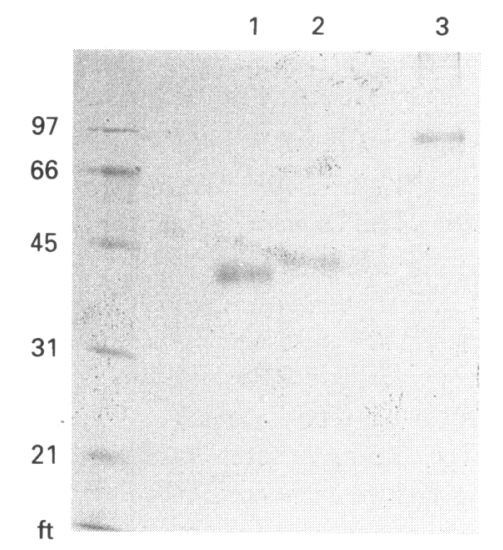

(c)

$\begin{array}{lllllllll}1 & 2 & 3 & 4 & 5 & 6 & 7 & 8 & 9\end{array}$

Fig. 1. Purification of glycoproteins from pig zona pellucida (ZP). Solubilized pig ZP was digested with endo- $\beta$-galactosidase and then fractionated on reverse-phase HPLC. (a) Elution profile of endo- $\beta$-galactosidase-digested PZP3 $\beta$ (E $\beta$ G-PZP3 $\beta$ ) (peak 1), E $\beta$ G-PZP3 $\alpha$ (peak 2) and E $\beta$ G-PZP2 (peak 3). Dashed line represents the acetonitrile gradient in $0.1 \%(\mathrm{v} / \mathrm{v})$ trifluoroacetic acid (b) SDS-PAGE (11\% gel) under nonreducing conditions of peak 1 (lane 1), peak 2 (lane 2) and peak 3 (lane 3 ) in (a). Molecular weight markers are indicated on left; ft: dye front. (c) SDS-PAGE (10\% gel) under nonreducing conditions of fractions on gel filtration column chromatography of solubilized ZP. The PZP2 fractions (lanes 2 and 3) and the PZP3 fractions (lanes 6, 7 and 8) were used in this study. Proteins were stained with Coomassie brilliant blue in both (b) and (c).

and then fractionated into endo- $\beta$-galactosidase-digested $\mathrm{PZP} 2$ (E $\beta$ G-PZP2), E $\beta G-P Z P 3 \alpha$ and E $\beta$ G-PZP $3 \beta$, by reverse-phase HPLC at $35^{\circ} \mathrm{C}$ on a Nucleosil $300-7 \mathrm{C} 18$ column $(4 \mathrm{~mm} \times 150 \mathrm{~mm})$ (Chemco Scientific Co., Ltd, Tokyo) with three steps of a linear gradient of acetonitrile (0-19 min: 0-37\%; $19-49 \mathrm{~min}: \quad 37-45 \%$; $49-54 \mathrm{~min}: \quad 45-80 \%$ ) in $0.1 \%(\mathrm{v} / \mathrm{v})$ trifluoroacetic acid at a flow rate of $1 \mathrm{ml} \mathrm{min}{ }^{-1}$. $E \beta G-P Z P 3 \beta, E \beta G-P Z P 3 \alpha$ and E $\beta$ G-PZP2 were eluted at concentrations of acetonitrile of about $38 \%, 40 \%$ and $60 \%$, respectively (Fig. 1a). PZP2 and PZP3 were purified from solubilized ZP by gel filtration chromatography as described by Nakano et al. (1990). The zona glycoproteins used in this study were homogeneous, as shown in Fig. Ib, c.

\section{Preparation of fluorescein isothiocyanate-labelled proteins}

Solubilized ZP, E $\beta$ G-PZP2, E $\beta$ G-PZP $3 \alpha$ or E $\beta$ G-PZP3 $\beta$ ( $0.5 \mathrm{mg}$ of each) was incubated with $400 \mathrm{nmol}$ fluorescein isothiocyanate $1^{-1}$ (FITC) at $25^{\circ} \mathrm{C}$ for $6 \mathrm{~h}$ in $1 \mathrm{ml}$ PBS (for solubilized ZP) or $115 \mathrm{mmol} \mathrm{NaHCO} \mathrm{I}^{-1}, \mathrm{pH} 8.5$ (for zona components). The reaction was stopped by the addition of glycine. Free FITC was removed by successive overnight dialyses against $10 \mathrm{mmol}$ sodium phosphate $1^{-1}$ and $300 \mathrm{mmol} \mathrm{NaCl} \mathrm{l^{-1 }}, \mathrm{pH} \mathrm{7.0,} \mathrm{and} \mathrm{then} 10 \mathrm{mmol}$ sodium phosphate $\mathrm{l}^{-1}$ and $150 \mathrm{mmol} \mathrm{NaCl} \mathrm{l}^{-1}, \mathrm{pH} 7.0$ and finally distilled water. FITC-labelled proteins were quantitated by amino acid analysis as described by Nakano et al. (1987). 
FITC-labelled proteins ( $10 \mu \mathrm{g}$ each) were extensively digested with pronase $(1 \mu \mathrm{g})$ in $0.1 \mathrm{ml} \mathrm{PBS}$ at $37^{\circ} \mathrm{C}$ for $18 \mathrm{~h}$. After adding $3 \mathrm{ml} 10 \mathrm{mmol}$ sodium phosphate $1^{-1}$ and $0.1 \%$ SDS, $\mathrm{pH} 7.0$, relative fluorescence intensity to free FITC of known concentration was measured at an emission wavelength of $515 \mathrm{~nm}$, with the excitation at $480 \mathrm{~nm}$. The molar ratios of FITC incorporated into solubilized ZP, E $\beta$ G-PZP2, E 3 GPZP $3 \alpha$ and E $\beta G-P Z P 3 \beta$ were estimated as $2.2,6.2,6.8$ and 4.0, respectively.

Cytochemical staining of boar spermatozoa with zona components and peanut agglutinin

Freshly ejaculated spermatozoa or cryopreserved epididymal spermatozoa were washed three times by centrifugation at $800 \mathrm{~g}$ for $3 \mathrm{~min}$ in $5 \mathrm{ml}$ of culture medium (medium A:

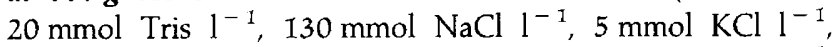
$1 \mathrm{mmol} \mathrm{MgCl}_{2} \mathrm{I}^{-1}, 3 \mathrm{mmol} \mathrm{CaCl} \mathrm{I}^{-1}, 5 \mathrm{mmol}$ glucose $\mathrm{I}^{-1}$, $3 \mathrm{mmol}$ sodium pyruvate $\left.\mathrm{I}^{-1}, 2 \%(\mathrm{w} / \mathrm{v}) \mathrm{BSA}, \mathrm{pH} 7.4\right)$ (Peterson et al., 1981) and incubated at $37^{\circ} \mathrm{C}$ for $1 \mathrm{~h}$ for capacitation (sperm concentrations were $10^{8}-10^{9} \mathrm{ml}^{-1}$ ). The spermatozoa were then incubated with $20 \mu \mathrm{g}$ FITC-labelled solubilized $\mathrm{ZP} \mathrm{ml} \mathrm{m}^{-1}$ or $50 \mu \mathrm{g}$ FITC-labelled zona components $\mathrm{ml}^{-1}$ dissolved in medium $\mathrm{A}$ for $1 \mathrm{~h}$ at $37^{\circ} \mathrm{C}$ in the presence or absence of $10 \mu \mathrm{g}$ rhodamine-conjugated peanut agglutinin (R-PNA) $\mathrm{ml}^{-1}$ (sperm concentration was $2 \times 10^{7} \mathrm{ml}^{-1}$ ). Spermatozoa were washed three times with medium A, fixed with $1.2 \%(\mathrm{w} / \mathrm{v})$ glutaraldehyde in $150 \mathrm{mmol}$ cacodylate $\mathrm{I}^{-1}, \mathrm{pH} 7.2$ (Shams-Borhan and Harrison, 1981) on a cover glass at room temperature for $50 \mathrm{~min}$, washed three times with medium A and mounted onto a slide glass. Samples were examined by phase contrast and fluorescence microscopy on a BH2-QRFL fluorescence microscope (Olympus, Tokyo). The effects of sulfated polysaccharides on the binding of solubilized ZP or EßGPZP $3 \alpha$ to spermatozoa were examined by preincubating spermatozoa in medium A containing $2 \mathrm{mg}$ fucoidan $\mathrm{ml}^{-1}$, $3.2 \mathrm{mg}$ dextran sulfate $\mathrm{ml}^{-1}$ or $10 \mathrm{mg}$ chondroitin sulfate $B$ $\mathrm{ml}^{-1}$ (Sigma, St Louis, MO) for $30 \mathrm{~min}$ at $37^{\circ} \mathrm{C}$ and then incubating with FITC-labelled solubilized ZP or FITC-labelled E $\beta G$-PZP3 $\alpha$. The concentrations of polysaccharides were considered to be adequate for the inhibition of the binding of FITC-labelled solubilized ZP to permeabilized spermatozoa (above $1 \mathrm{mg}$ fucoidan $\mathrm{ml}^{-1}$ and $1.5 \mathrm{mg}$ dextran sulfate $\mathrm{ml}^{-1}$; Jones, 1991) as was the concentration of chondroitin sulfate (above $10 \mathrm{mg} \mathrm{ml}^{-1}$; Jones, 1991) for $50 \%$ inhibition of binding of solubilized ZP to proacrosin.

Alternatively, capacitated spermatozoa were incubated with $80 \mu \mathrm{g}$ unmodified solubilized $\mathrm{ZP} \mathrm{ml}{ }^{-1}$ or $20 \mu \mathrm{g}$ each of unmodified zona components (PZP2, E $\beta$ G-PZP2, PZP3, E $\beta$ GPZP $3 \alpha$ and E $\beta$ G-PZP3 $\beta$ ) $\mathrm{ml}^{-1}$ instead of the FITC-labelled proteins described above. After washing and fixation, the cover glass was blocked with medium $\mathrm{A}$ at $37^{\circ} \mathrm{C}$ for $1 \mathrm{~h}$. Zona glycoproteins bound to spermatozoa were visualized using anti-pig ZP antiserum diluted to I:100 with medium $A$ as a first antibody and FITC-conjugated goat anti-rabbit $\operatorname{lgG}$ (Wako Pure Chemicals, Tokyo) diluted to 1:1000 as a second antibody. Anti-pig ZP antiserum recognizes all the components of pig ZP (Noguchi et al., 1994).
Detection of zona-binding protein in boar sperm extracts by using anti-pig $Z P$ antiserum or biotinylated $E \beta G-P Z P 3 a$

Cryopreserved epididymal spermatozoa were washed and capacitated as described above. The capacitated spermatozoa were washed with PBS once to remove BSA in medium A, suspended in SDS sample buffer (Laemmli, 1970) and heated at $100^{\circ} \mathrm{C}$ for $3 \mathrm{~min}$. After centrifugation at $2000 \mathrm{~g}$ for $3 \mathrm{~min}$, the soluble fraction ( $10^{4}$ spermatozoa per lane) was subjected to SDS-PAGE and then transferred to polyvinylidene difluoride (PVDF) membranes (Millipore, MA) as described by Towbin et al. (1979). The membranes were blocked with $3 \%$ BSA in Tris-buffered saline (TBS), incubated with $2 \mu \mathrm{g}$ solubilized ZP $\mathrm{ml}^{-1}$ or $1 \mu \mathrm{g}$ of each of the zona components $\mathrm{ml}^{-1}$ in TBS containing $1 \% \mathrm{BSA}$ for $3 \mathrm{~h}$ at $37^{\circ} \mathrm{C}$ and then washed three times (for 15 min each time) in TBS containing $0.05 \%$ Tween 20 (T-TBS). After incubation with anti-pig ZP antiserum diluted to $1: 200$ with TBS containing $1 \%$ BSA for $2 \mathrm{~h}$ at room temperature, the membranes were washed three times ( $15 \mathrm{~min}$ each) in T-TBS and then incubated with peroxidase conjugated anti-rabbit IgG (Wako Pure Chemicals, Tokyo) diluted to 1:1000. After washing, colour was developed with 4-chloro-1naphthol. Rabbit anti-boar acrosin antiserum (kindly donated by T. Baba, University of Tsukuba, Ibaraki) diluted to 1:1000 was used as a first antibody to detect proacrosin and acrosin.

E $\beta$ G-PZP $3 \alpha$ was biotinylated as described by TöpferPetersen and Henschen (1988) and tested as follows. Biotinylated E $\beta$ G-PZP $3 \alpha(0.3 \mu \mathrm{g})$ was subjected to SDS-PAGE followed by transfer to PVDF membrane. The membrane was blocked as described above and then incubated with streptavidin-peroxidase (Sigma, St Louis, MO) diluted to 1:1000 with TBS containing $1 \% \mathrm{BSA}(\mathrm{w} / \mathrm{v})$ at room temperature for $30 \mathrm{~min}$. After washing, colour was developed with 4-chloro-1-naphthol and biotinylated E $\beta$ G-PZP $3 \alpha$ was strongly stained. Acid extract from freshly ejaculated spermatozoa was prepared as described by Takada et al. (1994) and subjected to SDS-PAGE and then transferred to PVDF membrane. The membrane was blocked as described above, preincubated in the presence or absence of anti-acrosin antiserum diluted to 1:400 at room temperature for $1 \mathrm{~h}$. After washing three times ( $15 \mathrm{~min}$ each) with T-TBS, the membrane was incubated with $1 \mu \mathrm{g}$ biotinylated E $\beta$ G-PZP $3 \alpha \mathrm{ml}^{-1}$ in TBS containing $1 \%(\mathrm{w} / \mathrm{v}) \mathrm{BSA}$ at room temperature for $1 \mathrm{~h}$ and washed a further three times (15 min each) with T-TBS. Incubation with streptavidinperoxidase and colour development were performed as described above.

\section{Results}

\section{Evaluation of the acrosomal status of boar spermatozoa}

It was necessary to evaluate acrosomal status precisely so that the binding site of the $\mathrm{ZP}$ on spermatozoa could be examined. We evaluated acrosomal status by combining the fluorescent staining of spermatozoa with R-PNA (Jones, 1991) with the phase contrast observation of spermatozoa fixed with glutaraldehyde in cacodylate buffer (Shams-Borhan and 

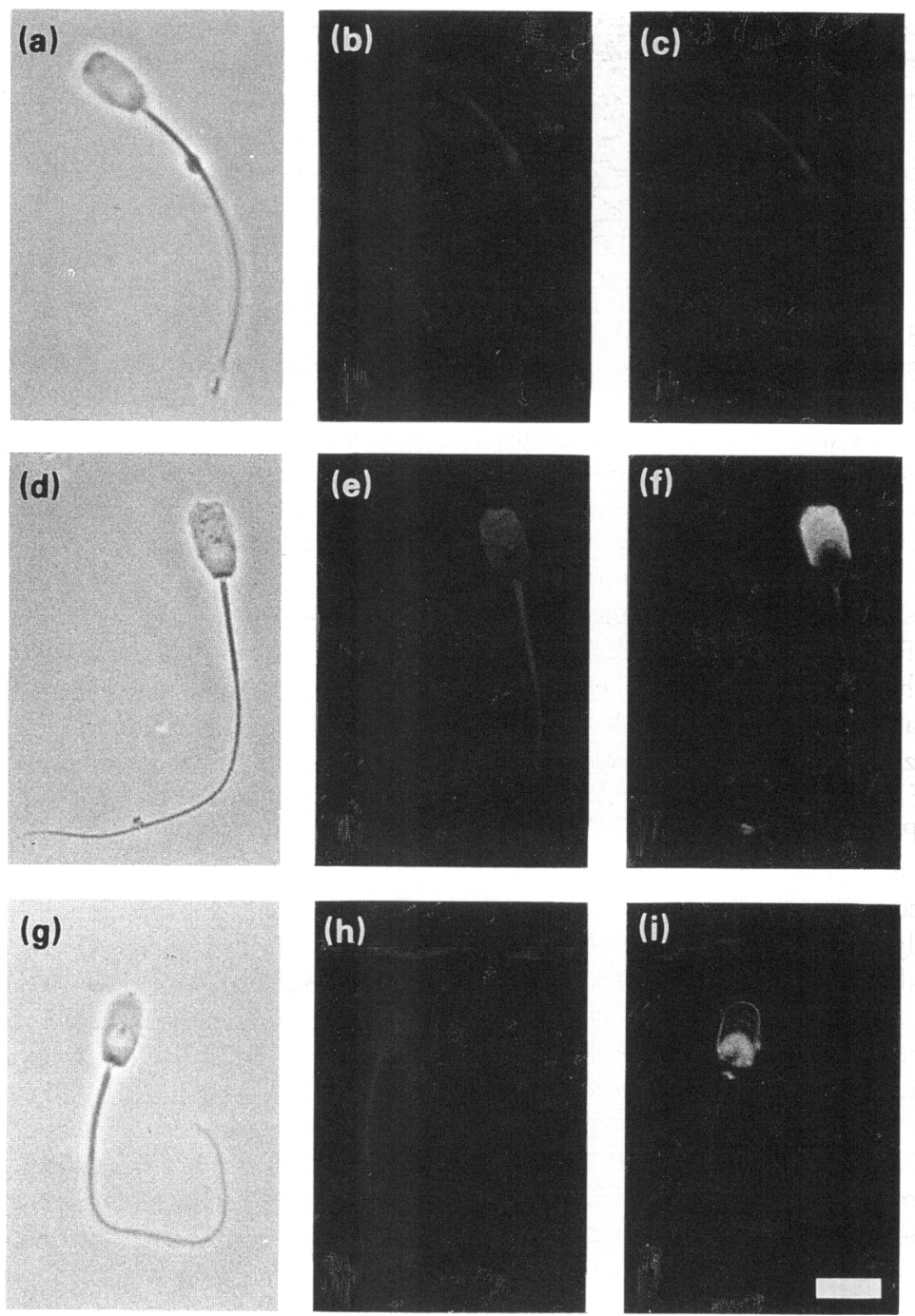

Fig. 2. Cytochemical staining of boar ejaculated spermatozoa with fluorescein isothiocyanate (FITC)-labelled solubilized zona pellucida (ZP) and rhodamineconjugated peanut agglutinin (R-PNA). Acrosome-intact ( $a, b$ and $c$ ), acrosomedamaged or partially acrosome-reacted ( $\mathrm{d}$, e and $\mathrm{f}$ ) and fully acrosome-reacted ( $\mathrm{g}$, $\mathrm{h}$ and i) spermatozoa were double stained with FITC-labelled solubilized ZP and R-PNA. The acrosomal status of the spermatozoa was evaluated by phase contrast image (a, d and $g$ ) and fluorescent staining pattern with R-PNA (c, f and i). Staining with FITC-labelled solubilized ZP is shown in (b), (e) and (h). Scale bar represents $10 \mu \mathrm{m}$.

Harrison, 1981). Acrosome-intact spermatozoa showed a clear line at the apex of the acrosome and were not stained with R-PNA (Fig. 2a, c). Acrosome-damaged or partially acrosomereacted spermatozoa showed a fuzzy, rippled or swollen acrosome and were stained with R-PNA on the acrosomal region (Fig. $2 \mathrm{~d}, \mathrm{f}$ ). The apical ridge of fully acrosome-reacted spermatozoa was absent and the acrosomal region of acrosome-reacted spermatozoa was not stained with R-PNA, but the equatorial segment was sometimes stained (Fig. $2 \mathrm{~g}$, i). After capacitation for $\mathrm{Ih}$, the acrosomes of about $70 \%$ of freshly ejaculated spermatozoa and $40 \%$ of cryopreserved epididymal spermatozoa were intact.
Analysis of the ZP-binding site on spermatozoa by cytochemical staining with solubilized ZP or ZP components

FITC-labelled solubilized ZP and FITC-labelled E $\beta$ G-PZP $3 \alpha$ inhibited the sperm-egg binding as effectively as unmodified solubilized ZP and ERG-PZP3 $\alpha$ in a competition assay performed in vitro as described by Noguchi et al. (1992).

FITC-labelled solubilized ZP bound to the acrosomal region only when the acrosomal region stained positively with R-PNA (Fig. 2e, f). The acrosome region of about $90 \%$ of acrosome-damaged or partially acrosome-reacted spermatozoa were stained with FITC-labelled solubilized ZP $(n=500)$. The 

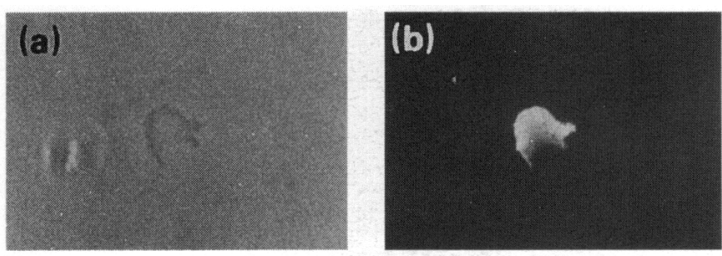

Fig. 3. Cytochemical staining of acrosomal cap with fluorescein isothiocyanate (FITC)-labelled solubilized zona pellucida (ZP). Acrosome reaction was induced by incubating ejaculated boar spermatozoa in medium A without BSA and containing $5 \mu \mathrm{mol}$ ionophore A23187 $\mathrm{I}^{-1}$ at $37^{\circ} \mathrm{C}$ for $\mathrm{I} \mathrm{h}$, and then incubated with FITC-labelled solubilized ZP. Most acrosomal caps were lost into the supernatant during washings, but several were found and observed. (a) Phase contrast, (b) fluorescent staining with FITC-labelled solubilized ZP.

percentage of acrosome-damaged or partially acrosome-reacted spermatozoa stained with FITC-labelled solubilized ZP and the fluorescence intensity in the acrosomal region were not changed in the absence of $\mathrm{Ca}^{2+}$ (data not shown). The staining of acrosome-intact spermatozoa with FITC-labelled solubilized $\mathrm{ZP}$ was as faint as that of spermatozoa incubated without FITC-labelled solubilized ZP (Fig. 2b). Staining with FITClabelled solubilized ZP was also observed in acrosomal caps released upon acrosome reaction (Fig. 3), but not on the inner acrosomal membrane of fully acrosome-reacted spermatozoa (Fig. 2h). When FITC-labelled fetuin or FITC-labelled IgG was incubated with capacitated spermatozoa as a control, the postacrosomal region and midpiece in some spermatozoa were faintly stained, but no significant staining was observed in the acrosomal region.

Approximately $92 \%$ of acrosome-damaged or partially acrosome-reacted spermatozoa were stained with FITC-labelled $\mathrm{E} \beta \mathrm{G}-\mathrm{PZP} 3 \alpha$ in the acrosomal region $(n=100)$ (Fig. 4e), whereas $33 \%$ and $19 \%$ of spermatozoa were stained with FITC-labelled E $\beta$ G-PZP2 and FITC-labelled E $\beta$ G-PZP $3 \beta$, respectively $(n=100)$, and the fluorescent intensities were much lower than that with FITC-labelled E $\beta$ G-PZP $3 \alpha$. Similar results were obtained by indirect immunofluorescent staining of sperm-bound zona glycoproteins using anti-pig ZP antiserum. In addition, the indirect immunofluorescent staining of sperm-bound PZP3 and PZP2 showed the same pattern on spermatozoa as E $\beta \mathrm{G}-\mathrm{PZP} 3 \alpha$ and E $\beta \mathrm{G}-\mathrm{PZP} 2$, respectively.

Acrosome-intact and fully acrosome-reacted spermatozoa were not stained with solubilized ZP or zona components when R-PNA was omitted from the reaction mixture, indicating that the inability of solubilized ZP or zona components to bind to acrosome-intact and fully acrosome-reacted spermatozoa is not due to the binding of peanut agglutinin to the oligosaccharides of zona glycoproteins or to sperm glycoproteins.

There were no differences in the binding sites of zona glycoproteins of freshly ejaculated and cryopreserved epididymal spermatozoa.

Effects of sulfated polysaccharides on the binding of FITC-labelled solubilized ZP, or FITC-labelled EBG-PZP3a, to the acrosome

The binding of FITC-labelled solubilized ZP to the acrosomal region of acrosome-damaged or partially acrosome- reacted spermatozoa was inhibited by fucoidan or dextran sulfate, competitors for the zona-binding of proacrosin, but very little by chondroitin sulfate B (Fig. 4a-d). The binding of FITC-labelled E $\beta$ G-PZP $3 \alpha$ to the acrosome was also inhibited by fucoidan or dextran sulfate but very little by chondroitin sulfate B (Fig. 4e-h).

\section{Proacrosin and acrosin-binding of E $\beta$ G-PZP3a}

The zona-binding proteins of capacitated epididymal spermatozoa were detected by immunostaining of zona glycoproteins with anti-pig ZP antiserum. Solubilized ZP, PZP3 and E $\beta$ G-PZP $3 \alpha$ bound to the sperm proteins of apparent molecular mass $56 \mathrm{kDa}, 52 \mathrm{kDa}, 42 \mathrm{kDa}$ and $38 \mathrm{kDa}$ (Fig. 5a, lanes 2, 4 and 5). Other than those proteins, E $\beta$ G-PZP $3 \alpha$ bound to $32 \mathrm{kDa}$ and $18 \mathrm{kDa}$ proteins (lane 5). E $\beta \mathrm{G}-\mathrm{PZP} 3 \beta$ did not show significant binding to sperm proteins (lane 7). PZP2 faintly recognized the $56 \mathrm{kDa}, 52 \mathrm{kDa}, 42 \mathrm{kDa}$ and $38 \mathrm{kDa}$ proteins (lane 8). The binding of solubilized ZP and E $\beta$ G-PZP $3 \alpha$ to the $56 \mathrm{kDa}, 52 \mathrm{kDa}, 42 \mathrm{kDa}$ and $38 \mathrm{kDa}$ proteins was reduced in the presence of $1.5 \mathrm{mg}$ fucoidan $\mathrm{ml}^{-1}$ (lanes 3 and 6). Moreover, anti-acrosin antiserum recognized the proteins with similar mobilities to the zona-binding proteins (lane 9). The effect of anti-acrosin antiserum on the binding of biotinylated E $\beta G-P Z P 3 \alpha$ to the sperm proteins was investigated to determine whether the zona-binding proteins are proacrosin and acrosin. Biotinylated ERG-PZP $3 \alpha$ bound to the $56 \mathrm{kDa}, 52 \mathrm{kDa}$ and $42 \mathrm{kDa}$ sperm proteins that were also detected by antiacrosin antiserum (Fig. 5b, lane 2) and to low molecular mass $(20-16 \mathrm{kDa})$ proteins in the acid extract from freshly ejaculated spermatozoa (lane 3). The binding of biotinylated E $\beta$ G.PZP $3 \alpha$ to the $56 \mathrm{kDa}, 52 \mathrm{kDa}$ and $42 \mathrm{kDa}$ proteins was inhibited by the preincubation of the membrane with anti-acrosin antiserum but the binding to the low molecular mass proteins was not inhibited (lane 4).

\section{Discussion}

It was found that solubilized $\mathrm{ZP}$ binds inside the acrosome and to the acrosomal cap released upon acrosome reaction. Binding of solubilized ZP to acrosome-intact or fully acrosome-reacted spermatozoa could not be detected by the methods used in the study reported here. Jones (1991) reported that FITC-labelled pig zona glycoproteins do not bind to intact spermatozoa but bind to the acrosomes of permeabilized spermatozoa and the results reported here are consistent with this. These findings suggest that the binding site of the $\mathrm{ZP}$ is localized mainly in the acrosomal matrix and on the membranous compartments in the acrosome.

Among the zona components, only E $\beta$ G-PZP $3 \alpha$ showed significant binding to the acrosome. The binding of solubilized $\mathrm{ZP}$ and E $\beta \mathrm{G}-\mathrm{PZP} 3 \alpha$ to the acrosome was inhibited by fucoidan and dextran sulfate. Western blot analyses of zona-binding proteins suggested that E $\beta$ G-PZP $\alpha$, as well as solubilized ZP, binds to proacrosin-acrosin, which is a major zona-binding protein of boar spermatozoa. Proacrosin-acrosin may mediate the binding of E $\beta G-P Z P 3 \alpha$, as well as that of solubilized $Z P$, to the acrosome of partially acrosome-reacted spermatozoa. Jones et al. (1988) proposed, taking the binding of proacrosin-acrosin 

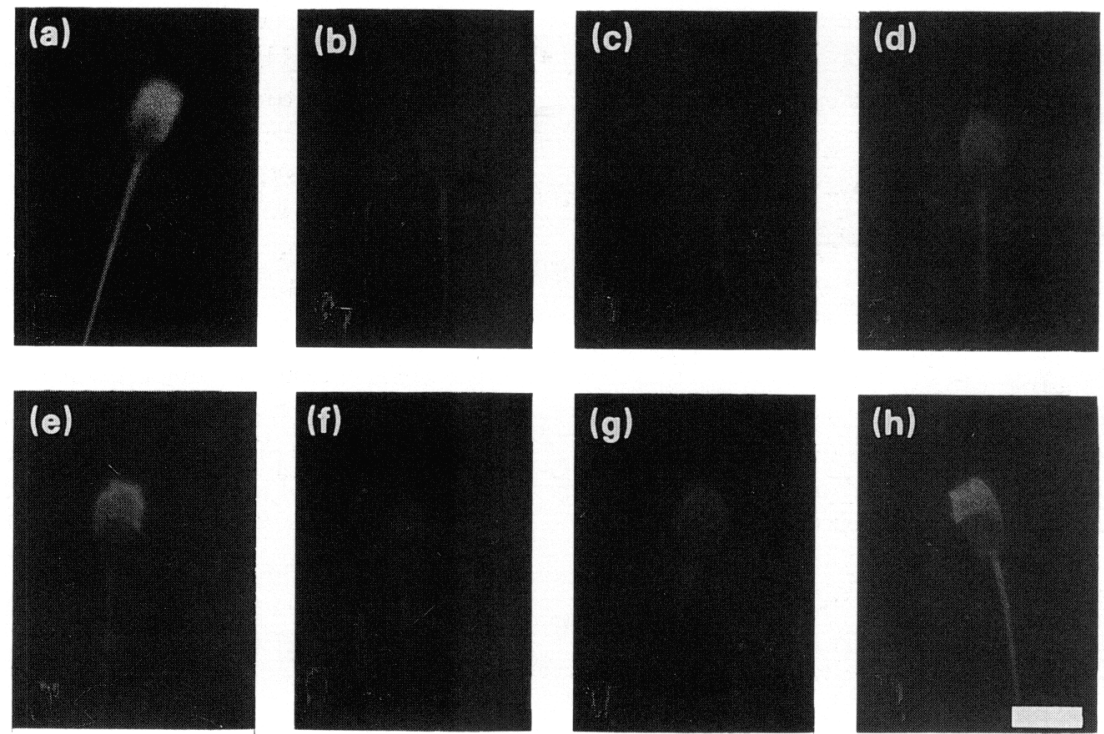

Fig. 4. Effects of sulfated polysaccharides on the staining of acrosome-damaged or partially acrosome-reacted spermatozoa with fluorescein isothiocyanate (FITC)-labelled solubilized zona pellucida (ZP) or FITC-labelled endo- $\beta$-galactosidase-digested PZP3 $\alpha$ (E $\beta$ G-PZP 3 $\alpha$ ). Spermatozoa were preincubated in the absence of competitors $(a, e)$ or in the presence of fucoidan $(b, f)$, dextran sulfate $(c, g)$ or chondroitin sulfate $B(d, h)$ and then stained with FITC-labelled solubilized ZP (a-d) or FITC-labelled E $\beta G-P Z P 3 \alpha(e-h)$. Scale bar represents $10 \mu \mathrm{m}$.

to $\mathrm{ZP}$ into consideration, that in pigs acrosome-intact spermatozoa or spermatozoa in the initial stage of acrosome reaction encounter the ZP. The spermatozoa in an intermediate state between acrosome-intact and fully acrosome-reacted bind firmly to the zona surface. Then, after the spermatozoa remove the acrosomal cap at the surface of the zona, the fully acrosome-reacted spermatozoa penetrate the zona matrix.

When pig eggs were incubated with capacitated spermatozoa and then washed vigorously by pipetting, acrosomal caps were left on the surface of the ZP (Yonezawa et al., unpublished).

A possible reason for the failure to detect the binding of solubilized ZP or E $\beta$ G-PZP3 $\alpha$ to acrosome-intact or fully acrosome-reacted spermatozoa is that the affinity of the solubilized form of the ZP for acrosome-intact and fully acrosomereacted spermatozoa is too low to detect the binding. Although solubilized pig ZP induces acrosome reaction in ejaculated boar spermatozoa, the increase in the number of acrosome-reacted spermatozoa is quite low in comparison with that in mice (Berger et al., 1989). We reported that native ZP structure is necessary for the completion of acrosome reaction in a fertilization assay in vitro (Yoshizawa et al., 1994). Native $\mathrm{ZP}$ structure might be necessary for the binding of the $\mathrm{ZP}$ to acrosome-intact spermatozoa.

We also showed that an inhibitor of $\beta-N$ acetylhexosaminidase reduces the rate of in vitro fertilization in pigs using cumulus-enclosed eggs, but that it changes the rate very little when cumulus-free eggs are used (Takada et al., 1994). $\beta$-N-acetylhexosaminidase purified from boar sperm acrosome possesses cumulus dispersion activity (Takada et al,, 1994). Although it is not yet clear whether acrosomal enzymes play an essential role in cumulus dispersion in pigs and the acrosomal status of fertilizing boar spermatozoa on the surface of $\mathrm{ZP}$ is not yet known, taking the involvement of proacrosinacrosinin sperm- $\mathrm{ZP}$ binding and the involvement of acrosomal enzymes in cumulus dispersion into consideration, we propose that some boar spermatozoa initiate the acrosome reaction before penetration into the cumulus oophorus and disperse cumulus cells by using their acrosomal enzymes, and that partially acrosome-reacted spermatozoa bind to the ZP and that this induces the completion of the acrosome reaction.

The apparent binding of E $\beta \mathrm{G}-\mathrm{PZP} 3 \alpha$, which does not contain sulfated $\mathrm{N}$-acetylpolylactosamine, to proacrosinacrosin suggests the involvement of a core region of sugar chains of PZP $3 \alpha$ other than sulfated $N$-acetylpolylactosamine in the binding of the ZP to proacrosin-acrosin. Noguchi et al. (1992) reported that neutral sugar chains isolated from PZP3 inhibit sperm-oocyte binding in a competition assay in vitro, whereas acidic sugar chains do not. Acidic carbohydrate chains of pig zona glycoproteins are sulfated at the C-6 position of GlcNAc residues in $\mathrm{N}$-acetyllactosamine (Noguchi and Nakano, 1992; Hokke et al., 1993). In the study reported here, a sulfated polymer of a neutral sugar, fucoidan and dextran sulfate, inhibited the binding of solubilized ZP or EßG-PZP $3 \alpha$ to the acrosome, whereas a sulfated polymer containing an amino sugar (chondroitin sulfate B) did not inhibit the binding. This finding suggests that the sulfated $\mathrm{N}$-acetylpolylactosamine of pig zona glycoproteins is not essential for the species-specific binding of the ZP to spermatozoa, and that if it is involved in binding, it has a subsidiary effect.

The inhibition of proacrosin-acrosin binding of E $\beta G-P Z P 3 \alpha$ by fucoidan may result from competition for common binding sites or the hindrance of access of E $\beta$ G-PZP $3 \alpha$ to its binding site on proacrosin-acrosin. Töpfer-Petersen et al. (1990) 
(a)

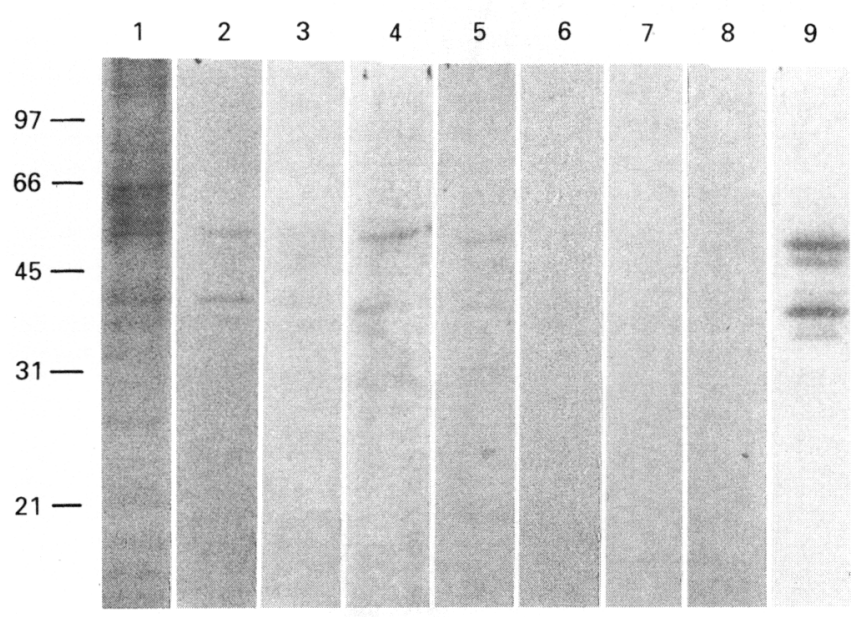

(b)

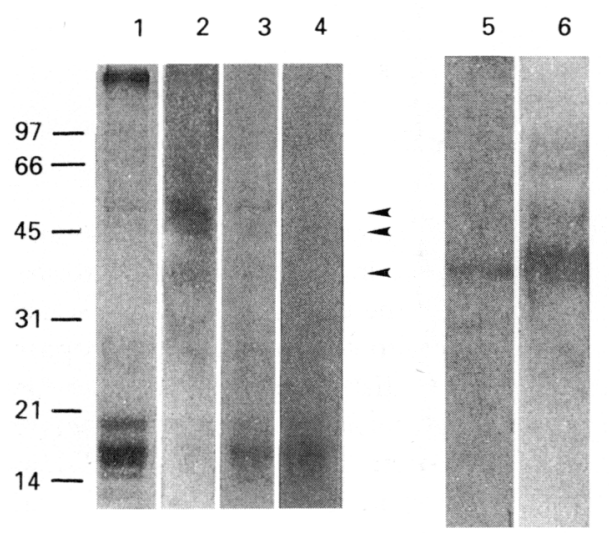

Fig. 5. Identification of zona-binding proteins in boar spermatozoa using (a) anti-pig zona pellucida (ZP) antiserum or (b) biotinylated endo- $\beta$-galactosidase-digested PZP $3 \alpha$ (E $\beta$ G-PZP $3 \alpha$ ). (a) Solubilized ZP (lanes 2 and 3), PZP3 (lane 4), E $\beta$ G-PZP3 $\alpha$ (lanes 5 and 6), E $\beta$ G-PZP3 $\beta$ (lane 7) or PZP2 (lane 8) were incubated with capacitated epididymal sperm proteins on polyvinylidene difluoride (PVDF) membranes and then the zona glycoproteins bound to sperm proteins were detected with anti-pig ZP antiserum. In lanes 3 and 6 , sperm proteins on PVDF membranes were incubated with solubilized ZP (3) or EßG-PZP3 $\alpha$ (6) in the presence of $1.5 \mathrm{mg}$ fucoidan $\mathrm{ml}^{-1}$. Proacrosin and acrosin were detected by anti-acrosin antiserum (lane 9). Anti-pig ZP antiserum and peroxidase-conjugated anti-rabbit IgG antibody did not stain sperm proteins (not shown). Lane 1, protein staining with Coomassie brilliant blue; molecular weight markers are indicated on the left. (b) Biotinylated E $\beta$ G-PZP $3 \alpha$ was incubated with acid extract from ejaculated spermatozoa on PVDF membranes preincubated in the absence (lane 3) or presence (lane 4) of anti-acrosin antiserum. Lane 1: Coomassie brilliant blue staining; lane 2: immunostaining of proacrosin and acrosin with anti-acrosin antiserum. Arrowheads on the right of lane 4 indicate the protein bands recognized with both anti-acrosin antiserum and biotinylated E $\beta$ G-PZP3 $\alpha$. Molecular weight markers are indicated on the left. Biotinylation of E $\beta$ G-PZP $3 \alpha$ was checked by western blot with streptavidin-peroxidase (lane 6). Lane 5: Coomassie brilliant blue staining of biotinylated E $\beta$ G-PZP $3 \alpha$. reported that the binding site of solubilized $\mathrm{ZP}$ was on the amino-terminal $15 \mathrm{kDa}$ part of the heavy chain. One of the important regulatory steps for species-specific recognition between gametes may be binding of ZP to proacrosin-acrosin as reported by Williams and Jones (1993). Studies on the molecular mechanism of binding of E $\beta$ G -PZP $3 \alpha$ to proacrosinacrosin would give further insight into the molecular basis of species-specific interaction of gametes.

The authors thank S. Miura and K. Sonohara. Chiba Prefectural Livestock Experiment Station, for kindly supplying the ejaculated boar spermatozoa; and K. Hozumi for technical assistance. This study was supported in part by a Saneyoshi Foundation and a Grant-in-Aid for Scientific Research from the Ministry of Education, Science and Culture of Japan.

\section{References}

Berger T, Turner KO, Meizel S and Hedrick JL (1989) Zona pellucida-induced acrosome reaction in boar sperm Biology of Reproduction 40 525-530

Hatanaka Y, Nagai T, Tobita T and Nakano M (1992) Changes in the properties and composition of zona pellucida of pigs during fertilization in vitro Journal of Reproduction and Fertility $95431-440$

Hedrick JL and Wardrip NJ (1987) On the macromolecular composition of the zona pellucida from porcine oocytes Developmental Biology 121 478-488

Hokke CH, Damm JBL, Kamerling JP and Vliegenthart JFG (1993) Structure of three acidic O-linked carbohydrate chains of porcine zona pellucida glycoproteins FEBS Letters 329 29-34

Jones R (1991) Interaction of zona pellucida glycoproteins, sulphated carbohydrates and synthetic polymers with proacrosin, the putative egg-binding protein from mammalian spermatozoa Development 111 1155-1163

Jones R, Brown CR and Lancaster RT (1988) Carbohydrate-binding properties of boar sperm proacrosin and assessment of its role in sperm-egg recognition and adhesion during fertilization Development 102 781-792

Laemmli UK (1970) Cleavage of structural proteins during the assembly of the head of bacteriophage T4 Nature 227 680-685

Liu DY and Baker HWG (1990) Inducing the human acrosome reaction with a calcium ionophore A23187 decreases sperm-zona pellucida binding with oocytes that failed to fertilize in vitro Journal of Reproduction and Fertility $\mathbf{8 9}$ $127-134$

Nakano M, Hatanaka Y, Sawai T, Kobayashi N and Tobita T (1987) Fractionation of glycoproteins from porcine zonae pellucidae into three families by high-performance liquid chromatography Biochemistry International 14, 417423

Nakano M, Hatanaka Y, Kobayashi N, Noguchi S, Ishikawa S and Tobita T (1990) Further fractionation of the glycoprotein families of porcine zona pellucida by anion-exchange HPLC and some characterization of the separated fractions Journal of Biochemistry (Tokyo) 107 144-150

Noguchi S and Nakano M (1992) Structure of the acidic N-linked carbohydrate chains of the 55-kDa glycoprotein family (PZP3) from porcine zona pellucida European Journal of Biochemistry $209883-894$

Noguchi S, Hatanaka Y, Tobita T and Nakano M (1992) Structural analysis of the $N$-linked carbohydrate chains of the $55-k \mathrm{Da}$ glycoprotein family (PZP3) from porcine zona pellucida European Journal of Biochemistry 204 1089-1100

Noguchi S, Yonezawa N, Katsumata T, Hashizume K, Kuwayama M, Hamano S, Watanabe S and Nakano M (1994) Characterization of the zona pellucida glycoproteins from bovine ovarian and fertilized eggs Biochinica of Biophysica Acta 1201 7-14

Peterson RN, Russell LD, Bundman D, Conway M and Freund M (1981) The interaction of living boar sperm and sperm plasma membrane vesicles with the porcine zona pellucida Developmental Biology 84 144-156

Sacco AG, Yurewicz EC, Subramanian MG and Matzat PD (1989) Porcine zona pellucida: association of sperm receptor activity with the $\alpha$-glycoprotein component (ZP3 $\alpha$ ) of the $\mathrm{Mr}=55000$ family (ZP3) Biology of Reproduction $41523-532$

Shams-Borhan G and Harrison RAP (1981) Production, characterization, and use of ionophore-induced, calcium-dependent acrosome reaction in ram spermatozoa Gamete Research 4 407-432 
Takada M, Yonezawa N, Yoshizawa M, Noguchi S, Hatanaka Y, Nagai T, Kikuchi K, Aoki $\mathbf{H}$ and Nakano $\mathbf{M}$ (1994) $\mathrm{pH}$-sensitive dissociation and association of $\beta-N$-acetylhexosaminidase from boar sperm acrosome Biology of Reproduction $50860-868$

Töpfer-Petersen E and Henschen A (1988) Zona pellucida-binding and fucosebinding of boar sperm acrosin is not correlated with proteolytic activity Biological Chemistry Hoppe-Seyler 369 69-75

Töper-Petersen E, Steinberger M, Ebner von Eschenbach C and Zucker A (1990) Zona pellucida-binding of boar sperm acrosin is associated with the $\mathrm{N}$-terminal peptide of the acrosin B-chain (heavy chain) FEBS Letters 265 51-54

Towbin H, Staehelin T and Gordon J (1979) Electrophoretic transfer of proteins from polyacrylamide gels to ritrocellulose sheets: procedure and some applications Proceedings of the National Academy of Sciences USA 764350 4354

Wassarman PM (1988) Zona pellucida glycoproteins Annual Review of Biochemistry $57415-442$
Williams RM and Jones R (1993) Specificity of binding of zona pellucida glycoproteins to sperm proacrosin and related proteins The Journal of Experimental Zoology 266 65-73

Yanagimachi R (1981) Mechanisms of fertilization in mammals. In Fertilization and Embryonic Development in vitro pp 81-182 Ed. L Mastroianni and JD Biggers. Plenum Press, New York

Yoshizawa M, Nagai T, Yonezawa N and Nakano M (1994) Native zona pellucida structure is required for completion of sperm acrosome reaction in porcine fertilization Theriogenology 41 1307-1313

Yurewicz EC, Sacco AG and Subramanian MG (1987) Structural characterization of the $\mathrm{Mr}=55000$ antigen $(\mathrm{ZP} 3)$ of porcine oocyte zona pellucida Journal of Biological Chemistry $262564-57 \mathrm{I}$

Yurewicz EC, Pack BA and Sacco AG (1992) Porcine oocyte zona pellucida $M_{\mathrm{r}} 55,000$ glycoproteins: identification of O-glycosylated domains Molecular Reproduction and Development 33 182-188 